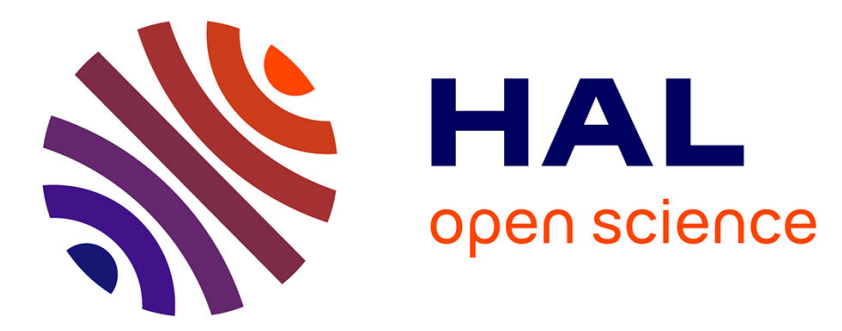

\title{
Conditional quantum logic using two atomic qubits
}

Igor E. Protsenko, Georges Reymond, Nicolas Schlosser, Philippe Grangier

\section{To cite this version:}

Igor E. Protsenko, Georges Reymond, Nicolas Schlosser, Philippe Grangier. Conditional quantum logic using two atomic qubits. Physical Review A : Atomic, molecular, and optical physics [19902015], 2002, 66(6), pp.062306. 10.1103/PhysRevA.66.062306 . hal-00509112

\section{HAL Id: hal-00509112 \\ https://hal.science/hal-00509112}

Submitted on 12 Apr 2016

HAL is a multi-disciplinary open access archive for the deposit and dissemination of scientific research documents, whether they are published or not. The documents may come from teaching and research institutions in France or abroad, or from public or private research centers.
L'archive ouverte pluridisciplinaire HAL, est destinée au dépôt et à la diffusion de documents scientifiques de niveau recherche, publiés ou non, émanant des établissements d'enseignement et de recherche français ou étrangers, des laboratoires publics ou privés. 


\title{
Conditional quantum logic using two atomic qubits
}

\author{
I. E. Protsenko, ${ }^{1,2,3}$ G. Reymond, ${ }^{1}$ N. Schlosser, ${ }^{1}$ and P. Grangier ${ }^{1}$ \\ ${ }^{1}$ Laboratoire Charles Fabry de l'Institut d'Optique, UMR 8501 du CNRS, F91403 Orsay, France \\ ${ }^{2}$ Lebedev Physics Institute, Leninsky Prospect 53, Moscow, Russia \\ ${ }^{3}$ Scientific Center of Applied Research, JINR, Dubna, Russia \\ (Received 2 June 2002; published 9 December 2002)
}

\begin{abstract}
In this paper we propose and analyze a feasible scheme where the detection of a single scattered photon from two trapped atoms or ions performs a conditional unitary operation on two qubits. As examples we consider the preparation of all four Bell's states, the reverse operation that is a Bell's measurement, and a controlled-NOT (CNOT) gate. We study the effect of atomic motion and multiple scattering by evaluating Bell's inequalities violations and by calculating the CNOT gate fidelity.
\end{abstract}

DOI: 10.1103/PhysRevA.66.062306

PACS number(s): 03.67.-a, 32.80.Pj, 03.65.Ud, 42.50.-p

\section{INTRODUCTION}

Implementing a quantum controlled-NOT (CNOT) gate is a key step in present attempts towards quantum computation. Many different schemes for CNOT gates using atoms or ions have been proposed [1-6], and some of them implemented [7-9]. These schemes generally require a strong quantum interaction between the particles that are used to carry the physical qubits. Though there are still many difficulties on the way, very promising results have been obtained recently using that approach, both with trapped ions [10] and neutral atoms [11].

Another possible way is to give up the requirement for a direct interaction between the qubits, and rather use an interference effect and a measurement-induced state projection to create the desired operation [12]. This provides "conditional" quantum gates, where the success of the logical operation is heralded by appropriate detection events. Under some conditions, such gates can also be exploited for fullfledged quantum computing [12].

Another type of conditional scheme was proposed in Refs. [13,14], for creating an entangled state of two atoms, simply by detecting a photon spontaneously emitted by the atom pair. This is done by avoiding to leave any "which path" information, so that the emitting atom cannot be identified. In such a scheme there is no direct interaction between the two atomic qubits, that can in principle be located very far from each other.

In this paper we propose to extend the ideas of Refs. $[13,14]$, to realize a full CNOT gate, or a Bell's-state measurement, or more generally to implement conditional unitary operations. Our scheme will be based on an experimental setup using two atoms in two neighboring microscopic dipole traps $[5,15,16]$, but it can be readily applied to other systems. In Sec. I we will describe how to realize a conditional unitary transformation that maps the four factorized states of two qubits onto the four maximally entangled Bell's states. Since a convenient experimental signature of entanglement is the violation of Bell's inequalities (BI) [17], we will evaluate the result of a test of BI on the "transformed" pair of qubits, taking into account imperfections due to the motion of atoms (Sec. II) and to the spontaneous emission of two photons by two atoms (Sec. III). BI measure- ments are studied quantitatively in Sec. IV. In Sec. V we describe a CNOT gate based on the Bell's states created by the procedure of Sec. I, and we calculate the fidelity of this gate, taking into account the motion of the atoms in the traps and the possible spontaneous emission of two photons. Finally we discuss these results and suggest developments of the proposed scheme.

\section{PREPARING FOUR ORTHOGONAL BELL'S STATES}

We consider two atoms $i=1,2$, trapped in two separate dipole traps and prepared in one of two states $|e\rangle_{i}$ or $|g\rangle_{i}$ of the ground-state hyperfine structure. We represent four initial states of the two-atom system as a vector column

$$
\{|\alpha \beta\rangle\}={ }^{t}\{|g g\rangle,|g e\rangle,|e g\rangle,|e e\rangle\}, \quad \alpha, \beta=e, g .
$$

Each atom can be excited to one of the upper states $\left|e^{\prime}\right\rangle_{i}$ or $\left|g^{\prime}\right\rangle_{i}$ by resonant $\sigma$-polarized laser fields of Rabi frequencies $\Omega_{g i}, \Omega_{e i}$, as shown in Fig. 1. The fields are weak, so that the probability to excite both atoms is much smaller than the probability to excite only one atom. An excited atom may emit spontaneously a photon, with the wave vector $\mathbf{k}$ and certain polarization, on $\pi$-polarized $\left|g^{\prime}\right\rangle_{i} \rightarrow|g\rangle_{i}$ or $\left|e^{\prime}\right\rangle_{i}$ $\rightarrow|e\rangle_{i}$ transitions. Occasionally, a photon passes through the optical system shown in Fig. 2 and it is registered by the photodetector. We assume that the polarizer $P$ transmits only $\pi$-polarized photons, and thus $\sigma$-polarized photons emitted on the $\left|e^{\prime}\right\rangle_{i} \rightarrow|g\rangle_{i}$ and $\left|g^{\prime}\right\rangle_{i} \rightarrow|e\rangle_{i}$ transitions will not be registered.

After the excitation, the wave function of the two atoms is changed from $|\alpha \beta\rangle$ to $\left|\Psi_{\alpha}\right\rangle_{1}\left|\Psi_{\beta}\right\rangle_{2}$,

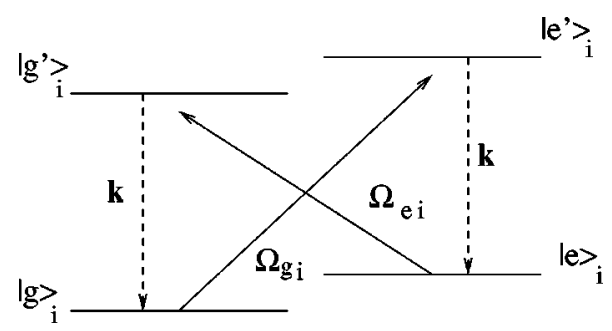

FIG. 1. Scheme of the relevant atom transitions. 


$$
\begin{gathered}
\left|\Psi_{e}\right\rangle_{i}=|e\rangle_{i}+b\left|g^{\prime}\right\rangle_{i} e^{i\left(\mathbf{k}_{e} \delta \mathbf{r}_{i}+\varphi_{e i}\right)}, \\
\left|\Psi_{g}\right\rangle_{i}=|g\rangle_{i}+b\left|e^{\prime}\right\rangle_{i} e^{\left(\mathbf{k}_{g} \delta \mathbf{r}_{i}+\varphi_{g i}\right)}, \\
\varphi_{\alpha i}=\mathbf{k}_{\alpha} \cdot \mathbf{r}_{i}+\varphi_{\alpha i}^{0},
\end{gathered}
$$

where $\delta \mathbf{r}_{i}$ describes fluctuations in the position of atom $i$ near the equilibrium due to the motion of the atom in the trap, $\mathbf{r}_{i}$ is the atom position at equilibrium, $\mathbf{k}_{e, g}$ are the wave vectors of the laser field resonant to either $e \rightarrow g^{\prime}$ or $g \rightarrow e^{\prime}$ transitions, $\varphi_{\alpha i}^{0}$ is the phase of the laser field $\Omega_{\alpha i}, b \ll 1$ is a real constant.

The registration of a photon means that the wave function $\left|\Psi_{\alpha}\right\rangle\left|\Psi_{\beta}\right\rangle$ is projected to a Bell's state $\left|B_{\alpha \beta}\right\rangle=|\mathbf{k}\rangle \hat{B}|\alpha \beta\rangle$, where $|\mathbf{k}\rangle$ is the state of the field with one spontaneously emitted photon. For example, $\left|\Psi_{g}\right\rangle\left|\Psi_{g}\right\rangle$ is projected to

$$
\begin{gathered}
|\mathbf{k}\rangle \hat{B}|g g\rangle=|\mathbf{k}\rangle\left|B_{g g}\right\rangle \\
\left|B_{g g}\right\rangle=(1 / \sqrt{2})\left\{|g e\rangle e^{i\left[\mathbf{q}_{g} \delta \mathbf{r}_{2}+k l_{2}(\mathbf{k})+\varphi_{g 2}\right]}\right. \\
\left.+|e g\rangle e^{i\left[\mathbf{q}_{g} \delta \mathbf{r}_{1}+k l_{1}(\mathbf{k})+\varphi_{g 1}\right]}\right\}
\end{gathered}
$$

where $\mathbf{q}_{\alpha}=\mathbf{k}_{\alpha}-\mathbf{k}, k=|\mathbf{k}|$, and $l_{i}(\mathbf{k})$ is the optical length which a photon travels through the optical system towards the photodetector. The optical system is set in such a way that the images $1^{\prime}, 2^{\prime}$ of atoms 1 and 2 perfectly coincide on the photodetector. This means that $l_{i}(\mathbf{k})$ is the same for all registered photons, and therefore $k l_{i}(\mathbf{k})=k l_{i}$. Introducing the vector column $\left\{\left|B_{\alpha \beta}\right\rangle\right\}={ }^{t}\left\{\left|B_{g g}\right\rangle,\left|B_{g e}\right\rangle,\left|B_{e g}\right\rangle,\left|B_{e e}\right\rangle\right\}$ of the Bell's states we can express them in terms of the initial states (1) as $\left\{\left|B_{\alpha \beta}\right\rangle\right\}=[B]^{\prime}\{|\alpha \beta\rangle\}$, where

$$
[B]^{\prime}=\frac{1}{\sqrt{2}}\left[\begin{array}{cccc}
0 & e^{i\left(\mathbf{q}_{g} \delta \mathbf{r}_{2}+\varphi_{g 2}+k l_{2}\right)} & e^{i\left(\mathbf{q}_{g} \delta \mathbf{r}_{1}+\varphi_{g 1}+k l_{1}\right)} & 0 \\
e^{i\left(\mathbf{q}_{e} \delta \mathbf{r}_{2}+\varphi_{e 2}+k l_{2}\right)} & 0 & 0 & e^{i\left(\mathbf{q}_{g} \delta \mathbf{r}_{1}+\varphi_{g 1}+k l_{1}\right)} \\
e^{i\left(\mathbf{q}_{e} \delta \mathbf{r}_{1}+\varphi_{e 1}+k l_{1}\right)} & 0 & 0 & e^{i\left(\mathbf{q}_{g} \delta \mathbf{r}_{2}+\varphi_{g 2}+k l_{2}\right)} \\
0 & e^{i\left(\mathbf{q}_{e} \delta \mathbf{r}_{1}+\varphi_{e 1}+k l_{1}\right)} & e^{i\left(\mathbf{q}_{e} \delta \mathbf{r}_{2}+\varphi_{e 2}+k l_{2}\right)} & 0
\end{array}\right]
$$

is a matrix of Bell's operator $\hat{B}$.

In general, the wave function $\left|B_{g g}\right\rangle\left(\left|B_{g e}\right\rangle\right)$ is not orthogonal to $\left|B_{e e}\right\rangle\left(\left|B_{e g}\right\rangle\right)$. In order to make sure that all Bell's states are orthogonal, one has to satisfy two conditions,

$$
\begin{aligned}
0= & \left\langle B_{e e} \mid B_{g g}\right\rangle=W_{g e} e^{i\left(\varphi_{g 1}-\varphi_{e 2}+k l_{1}-k l_{2}\right)} \\
& +W_{e g}^{*} e^{i\left(\varphi_{g 2}-\varphi_{e 1}+k l_{2}-k l_{1}\right)}
\end{aligned}
$$

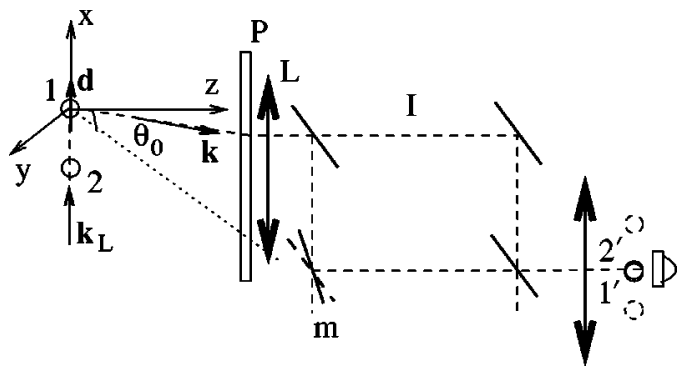

FIG. 2. Proposed scheme for conditional quantum logic. Atoms 1,2 are placed in a focal plane of the input lens $L$ of the optical system. The atom pair is excited by the laser field with the wave vector $\mathbf{k}_{L}$, circularly polarized in the $y z$ plane, and emits a photon with the wave vector $\mathbf{k}$ on the $x$-polarized transition. The polarizer $P$ selects $x$-polarized photons that are transmitted through an interferometer $I$ towards a photodetector. A mirror $m$ of the interferometer is tilted so that the images $1^{\prime}, 2^{\prime}$ of the two atoms coincide on the photodetector. The unit vector in the direction of a $\pi$-polarized atomic dipole is denoted as $\mathbf{d}$, and $\theta_{0}$ is the aperture angle of the lens $L$.

$$
\begin{aligned}
0= & \left\langle B_{e g} \mid B_{g e}\right\rangle=W_{g g} e^{i\left(\varphi_{g 1}-\varphi_{g 2}+k l_{1}-k l_{2}\right)} \\
& +W_{e e}^{*} e^{i\left(\varphi_{e 2}-\varphi_{e 1}+k l_{2}-k l_{1}\right)}
\end{aligned}
$$

where $W_{\alpha \beta}=e^{i\left(\mathbf{q}_{\alpha} \delta \mathbf{r}_{1}-\mathbf{q}_{\beta} \delta \mathbf{r}_{2}\right)}$. If the atoms are very cold in a steep trap, so that they are deeply in the Lamb-Dicke regime, one should take $W_{\alpha \beta}=1$ in Eqs. (5) and (6). But we point out that the resulting conditions are actually independent of the atoms motion. Indeed,

$$
\begin{aligned}
& \left\langle W_{\alpha \beta}\right\rangle=\left\langle W_{\alpha \beta}^{*}\right\rangle=V_{\alpha} V_{\beta} \equiv 1-D(T), \\
& V_{\alpha}=\left\langle\sum_{n=0}^{-\infty} \frac{(-1)^{n}}{(2 n) !}\left\langle\left(\mathbf{q}_{\alpha} \cdot \delta \mathbf{r}\right)^{2 n}\right\rangle_{T}\right\rangle_{\mathbf{q}_{\alpha}},
\end{aligned}
$$

where $\langle\cdots\rangle_{T}$ and $\langle\cdots\rangle_{\mathbf{q}_{\alpha}}$ mean, respectively, the average over the atom motion and over directions of registered photons. We average separately over symmetrical and statistically independent motion of each atom, drop index $i$ in $\delta \mathbf{r}_{i}$, and introduce the parameter $D(T), 0 \leqslant D(T)<1$, where $T$ is the temperature associated with the random motion of the atoms. One can see that $\left\langle W_{\alpha \beta}\right\rangle$ and $\left\langle W_{\alpha \beta}^{*}\right\rangle$ disappear from orthogonality conditions (5),(6), which are reduced to a single condition

$$
\varphi_{g 2}^{0}-\varphi_{g 1}^{0}+\varphi_{e 2}^{0}-\varphi_{e 1}^{0}+\left(\mathbf{k}_{e}+\mathbf{k}_{g}\right)\left(\mathbf{r}_{2}-\mathbf{r}_{1}\right)+2 k\left(l_{2}-l_{1}\right)=\pi .
$$

In our geometry we have $\varphi_{\alpha 1}^{0}=\varphi_{\alpha 2}^{0}$, and thus this condition becomes

$$
\left(\mathbf{k}_{e}+\mathbf{k}_{g}\right)\left(\mathbf{r}_{2}-\mathbf{r}_{1}\right)+2 k\left(l_{2}-l_{1}\right)=\pi
$$


There are various ways to fulfill this condition. If one chooses $\mathbf{k}_{e}=-\mathbf{k}_{g}$, i.e., the $\sigma_{+}$and $\sigma_{-}$lasers are propagating in opposite directions, the condition for orthogonality of the Bell's states is obtained by adjusting the interferometer path difference so that $k\left(l_{2}-l_{1}\right)=\pi / 2$. But it is also possible to take $\mathbf{k}_{e}=\mathbf{k}_{g}=\mathbf{k}_{L}$, together with $\mathbf{k}_{L}\left(\mathbf{r}_{2}-\mathbf{r}_{1}\right)=\pi / 2$, obtained by adjusting the trap's positions. Assuming then that $k l_{2}=k l_{1}=2 n \pi$ for simplicity, taking (here and everywhere below) the origin of the coordinate system on the atom 1 and defining $\mathbf{q}=\mathbf{k}_{L}-\mathbf{k}$, the Bell's operator matrix can be written,

$$
[B]^{\prime}=\frac{1}{\sqrt{2}}\left[\begin{array}{cccc}
0 & i e^{i \mathbf{q} \delta \mathbf{r}_{2}} & e^{i \mathbf{q} \delta \mathbf{r}_{1}} & 0 \\
i e^{i \mathbf{q} \delta \mathbf{r}_{2}} & 0 & 0 & e^{i \mathbf{q} \delta \mathbf{r}_{1}} \\
e^{i \mathbf{q} \delta \mathbf{r}_{1}} & 0 & 0 & i e^{i \mathbf{q} \delta \mathbf{r}_{2}} \\
0 & e^{i \mathbf{q} \delta \mathbf{r}_{1}} & i e^{i \mathbf{q} \delta \mathbf{r}_{2}} & 0
\end{array}\right],
$$

which converts four initial atom states to four Bell's states, which are orthogonal in average over the atom motion. Though the condition for the orthogonality in average depends only on the atoms equilibrium positions, the final fidelity of the conditional unitary transformation will obviously depend on the atoms motion, due to the $\delta \mathbf{r}_{1}$ and $\delta \mathbf{r}_{2}$ in the $[B]^{\prime}$ matrix.

In order to simplify the local operations used in the rest of the paper, it is convenient to perform two phase transformations for atom 2, that make the change $|e\rangle_{2} \rightarrow-i|e\rangle_{2}$ just before the photon observation and $|e\rangle_{2} \rightarrow i|e\rangle_{2}$ right after it. Taking into account such transfornations as $\operatorname{diag}\{1,-i, 1$ $-i\}[B]^{\prime} \operatorname{diag}\{1, i, 1, i\} \equiv[B]$, where diag means diagonal matrix, we find

$$
[B]=\frac{1}{\sqrt{2}}\left[\begin{array}{cccc}
0 & -e^{i \mathbf{q} \delta \mathbf{r}_{2}} & e^{i \mathbf{q} \delta \mathbf{r}_{1}} & 0 \\
e^{i \mathbf{q} \delta \mathbf{r}_{2}} & 0 & 0 & e^{i \mathbf{q} \delta \mathbf{r}_{1}} \\
e^{i \mathbf{q} \delta \mathbf{r}_{1}} & 0 & 0 & -e^{i \mathbf{q} \delta \mathbf{r}_{2}} \\
0 & e^{i \mathbf{q} \delta \mathbf{r}_{1}} & e^{i \mathbf{q} \delta \mathbf{r}_{2}} & 0
\end{array}\right],
$$

which has real elements in the absence of atom motion $\delta \mathbf{r}_{i}$ $=0$. In a geometry where the phases $\varphi_{\alpha i}^{0}$ can be independently controlled, one can obtain the matrix (10) more straightforwardly, for example by choosing in Eq. (8) $k l_{2}$ $=k l_{1}=2 n \pi, \mathbf{k}_{e}=\mathbf{k}_{g}=\mathbf{k}_{L}, \mathbf{r}_{1}=0$, and

$$
\varphi_{g 1}^{0}=\varphi_{e 1}^{0}=0, \quad \varphi_{e 2}^{0}=-\mathbf{k}_{L} \cdot \mathbf{r}_{2}, \quad \varphi_{g 2}^{0}=\pi-\mathbf{k}_{L} \cdot \mathbf{r}_{2} .
$$

Below we refer to $[B]$ as a Bell's operator matrix supposing either that condition (9) is true and the Bell's operation is the photon observation procedure with the two phase transformations for atom 2, or that there is only the photon observation, but conditions (11) are satisfied.

In order to get a physical understanding about the quality of the Bell's-states preparation, we will now look in detail whether the prepared states can violate Bell's inequalities. In these calculations we will use the expression (10) corresponding to $\mathbf{k}_{e}=\mathbf{k}_{g}=\mathbf{k}_{L}$, but similar results could be easily obtained in the case where $\mathbf{k}_{e}=-\mathbf{k}_{g}$ (the fully phasematched situation where the atoms' positions would cancel out is not accessible with our experimental geometry).

\section{BELL'S INEQUALITIES}

When the atoms are prepared in a Bell's state, the statistical behavior of measurable quantities (such as the population of state $|\alpha\rangle_{i}$ ) is governed by the entangled wave function $\left|B_{\alpha \beta}\right\rangle$. Here BI will be used as a simple experimental characterization of the degree of entanglement of the atom pair. As we will see below, either atoms motion or simultaneous excitation of the two atoms may reduce or even suppress the BI violation.

In order to test BI we carry out the following sequence of operations.

(1) The atoms are prepared in one of the states (1).

(2) Atoms are excited by weak laser pulses under the conditions of Eq. (9).

(3) One spontaneously emitted photon is registered. If there is no photon after some delay, the stages (1),(2) are repeated until one photon is registered.

(4) Raman transitions for each atom are carried out so that

$$
\begin{gathered}
|g\rangle_{i} \rightarrow \cos \left(\theta_{i}\right)|g\rangle_{i}-\sin \left(\theta_{i}\right)|e\rangle_{i}, \\
|e\rangle_{i} \rightarrow \cos \left(\theta_{i}\right)|e\rangle_{i}+\sin \left(\theta_{i}\right)|g\rangle_{i} .
\end{gathered}
$$

(5) Populations of $|\alpha\rangle_{i}$ states are measured.

(6) Operations (1)-(5) have to be repeated until a full statistical ensemble of results for the population of $|\alpha\rangle_{i}$ states is obtained.

(7) The steps (1)-(6) are repeated for four different Raman transitions with four pairs of angles $\left\{\theta_{1}, \theta_{2}\right\},\left\{\theta_{1}^{\prime}, \theta_{2}^{\prime}\right\}$, $\left\{\theta_{1}^{\prime}, \theta_{2}\right\}$, and $\left\{\theta_{1}, \theta_{2}^{\prime}\right\}$.

After the operations (1)-(6) are carried out, the state of atoms and a photon is $|\mathbf{k}\rangle \hat{R} \hat{B}|\alpha \beta\rangle$, where the operator $\hat{R}$ describes Raman transitions (12) for two atoms. We note that the wave function is written on the right to the operator. We preserve the same convention for matrix notations, so that $\hat{R} \hat{B}|\alpha \beta\rangle$ is an element of $[R B]\{|\alpha \beta\rangle\}$, where $\{|\alpha \beta\rangle\}$ is the vector column (1). It is shown in Appendix B that in this case the matrix $[R B]$ of $\hat{R} \hat{B}$ operator is the matrix product $[B]\left[R\left(\theta_{i}\right)\right]$; the matrix $\left[R\left(\theta_{i}\right)\right]$ for the Raman transitions is given by Eq. (B2) of Appendix B, and $[B]$ is given by Eq. (10). Here and below we denote the dependence on $\theta_{1}$ and $\theta_{2}$ as a dependence on $\theta_{i}$, when it does not lead to confusion.

Let us call $P_{\alpha \beta}^{(\gamma \delta)}\left(\theta_{i}\right)$ the probability to find atoms in state $|\gamma \delta\rangle$, while the initial atom state is $|\alpha \beta\rangle$. By taking the modulus square of each matrix element in $[R B]$ one can find

$$
\begin{aligned}
P_{g g}^{(g g)}\left(\theta_{i}\right) & =P_{g e}^{(g e)}\left(\theta_{i}\right)=P_{g e}^{(e g)}\left(\theta_{i}\right)=P_{g g}^{(e e)}\left(\theta_{i}\right) \\
& =0.5\left[\sin ^{2}\left(\theta_{1}-\theta_{2}\right)+0.5 Q\left(T, \theta_{i}\right)\right], \\
P_{g e}^{(g g)}\left(\theta_{i}\right) & =P_{g g}^{(g e)}\left(\theta_{i}\right)=P_{g g}^{(e g)}\left(\theta_{i}\right)=P_{g e}^{(e e)}\left(\theta_{i}\right) \\
& =0.5\left[\cos ^{2}\left(\theta_{1}-\theta_{2}\right)-0.5 Q\left(T, \theta_{i}\right)\right], \\
P_{e g}^{(g g)}\left(\theta_{i}\right) & =P_{e e}^{(g e)}\left(\theta_{i}\right)=P_{e e}^{(e g)}\left(\theta_{i}\right)=P_{e g}^{(e e)}\left(\theta_{i}\right) \\
& =0.5\left[\cos ^{2}\left(\theta_{1}+\theta_{2}\right)+0.5 Q\left(T, \theta_{i}\right)\right],
\end{aligned}
$$




$$
\begin{aligned}
P_{e e}^{(g g)}\left(\theta_{i}\right) & =P_{e g}^{(g e)}\left(\theta_{i}\right)=P_{e g}^{(e g)}\left(\theta_{i}\right)=P_{e e}^{(e e)}\left(\theta_{i}\right) \\
& =0.5\left[\sin ^{2}\left(\theta_{1}+\theta_{2}\right)-0.5 Q\left(T, \theta_{i}\right)\right],
\end{aligned}
$$

where $Q\left(T, \theta_{i}\right)=D(T) \sin \left(2 \theta_{1}\right) \sin \left(2 \theta_{2}\right)$ and $D(T)$ is given by Eq. (7). One has $D(T)=0$ in the absence of atoms motion, in this case the maximum violation of Bell's inequalities is obtained. In the opposite (high-temperature) situation, where $D(T) \rightarrow 1$, one obtains from Eqs. (13),

$$
P_{g g}^{(g g)}\left(\theta_{i}\right) \rightarrow 0.5\left[\sin ^{2}\left(\theta_{1}\right) \cos ^{2}\left(\theta_{2}\right)+\sin ^{2}\left(\theta_{2}\right) \cos ^{2}\left(\theta_{1}\right)\right],
$$

and similar expressions for the other probabilities. This corresponds to a "classical" limit where BI cannot be violated. Thus $D(T)$ is a "decoherence parameter" which grows up with the temperature from $D(0)=0$ to $\max D=1$.

For each atom $i=1,2$ we define a random variable $\xi_{i}$, with values +1 or -1 depending on whether an atom is found, respectively, in $|g\rangle_{i}$ or $|e\rangle_{i}$ state after registering a photon and carrying out the Raman transition. With the help of Eqs. (13) one can find $\left\langle\xi_{1}\right\rangle=\left\langle\xi_{2}\right\rangle=0,\left\langle\xi_{1}^{2}\right\rangle=\left\langle\xi_{2}^{2}\right\rangle=1$, where the average is made over the results of a sequence of operations (1)-(6). The correlation functions are given by

$$
\begin{aligned}
E_{\alpha \beta}\left(\theta_{i}\right) & \equiv \frac{\left\langle\xi_{1} \xi_{2}\right\rangle-\left\langle\xi_{1}\right\rangle\left\langle\xi_{2}\right\rangle}{\sqrt{\left\langle\xi_{1}^{2}\right\rangle\left\langle\xi_{2}^{2}\right\rangle}}=\left\langle\xi_{1} \xi_{2}\right\rangle \\
& =2\left[P_{\alpha \beta}^{(e e)}\left(\theta_{i}\right)-P_{\alpha \beta}^{(e g)}\left(\theta_{i}\right)\right],
\end{aligned}
$$

so that

$$
\begin{aligned}
& E_{g e}\left(\theta_{i}\right)=-E_{g g}\left(\theta_{i}\right)=\cos \left[2\left(\theta_{1}-\theta_{2}\right)\right]-Q\left(T, \theta_{i}\right), \\
& E_{e g}\left(\theta_{i}\right)=-E_{e e}\left(\theta_{i}\right)=\cos \left[2\left(\theta_{1}+\theta_{2}\right)\right]+Q\left(T, \theta_{i}\right) .
\end{aligned}
$$

As usual we define the quantity

$$
S_{\alpha \beta}\left(\theta_{i}, \theta_{i}^{\prime}\right)=E_{\alpha \beta}\left(\theta_{i}\right)-E_{\alpha \beta}\left(\theta_{1} \theta_{2}^{\prime}\right)+E_{\alpha \beta}\left(\theta_{1}^{\prime} \theta_{2}\right)+E_{\alpha \beta}\left(\theta_{i}^{\prime}\right)
$$

for each initial state $|\alpha, \beta\rangle$, then the Bell's inequalities read [18]

$$
-2 \leqslant S_{\alpha \beta}\left(\theta_{i}, \theta_{i}^{\prime}\right) \leqslant 2 .
$$

The results (13)-(16) are similar to ones obtained for the BI test with polarization-entangled photon pairs [19], the difference is that here the decoherence is taken into account by means of $D(T)$. In the following section we look for the violation of inequalities (17) for each initial state of the twoatom system.

\section{EFFECT OF ATOM MOTION ON BELL'S INEQUALITIES TEST}

In order to predict accurately the value of $S_{\alpha \beta}\left(\theta_{i}, \theta_{i}^{\prime}\right)$ we have to calculate the factor $D(T)$, which depends on the trapping potential and the aperture angle $\theta_{0}$ of the input lens of the optical system. In general, the trapping potential is anharmonic, nonsymmetric and $\theta_{0}$ is not small. All of these

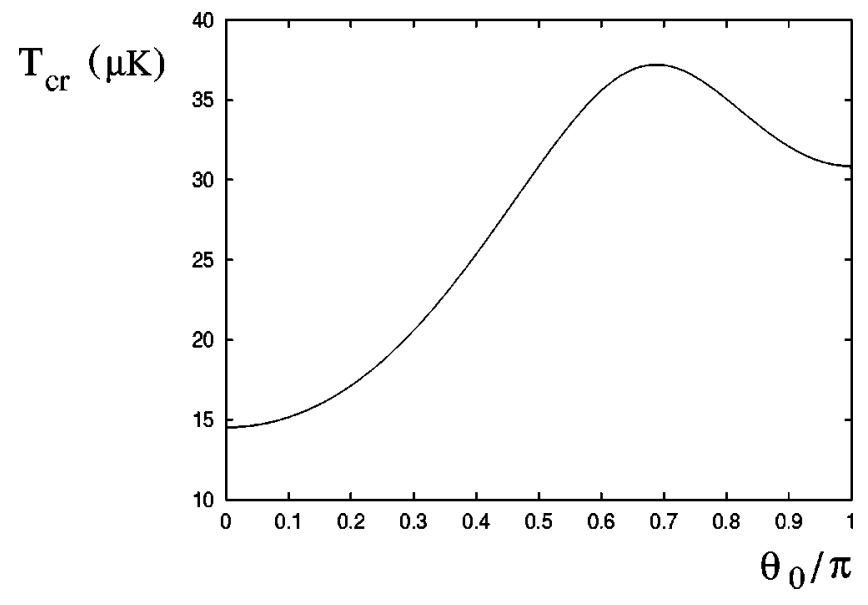

FIG. 3. Critical temperature as a function of the aperture angle of the optical system.

complicate the precise calculation of $D(T)$, which will be carried out elsewhere. Here we will consider a simple orderof-magnitude estimation, using anharmonic approximation for the trapping potential. The procedure carried out in Appendix A (see also Ref. [20]) leads to

$$
D(T) \approx 1-e^{-T / T_{c r}}, \quad k_{B} T_{c r}=\frac{h^{2} \nu_{e f f}^{2}}{2 E_{R}},
$$

where $T_{c r}$ is a critical temperature such that $D\left(T>T_{c r}\right)$ $\approx 1, \nu_{\text {eff }}$ is an effective frequency of atom motion in the trap. For the aperture angle $\theta_{0}=\pi / 4$, as it is in our case, $\nu_{e f f}^{-2} \approx 1.25 \nu_{\perp}^{-2}+0.75 \nu_{\|}^{-2}$, where $\nu_{\perp}$ and $\nu_{\|}$are the frequencies of the motion of atoms in $x, y$, and in $z$ directions, respectively; $E_{R}=\hbar^{2} k^{2} /\left(2 m_{a t}\right) \equiv h \nu_{R}$ is the recoil energy, and $k_{B}$ is the Boltzmann constant. Using $\nu_{R}=3.6 \mathrm{kHz}$ for $\mathrm{Rb}^{87}$ atoms and our estimations $\nu_{\perp}=200 \mathrm{kHz}$ and $\nu_{\|}$ $=50 \mathrm{kHz}$, we obtain $\nu_{e f f}=55 \mathrm{kHz}$ and $T_{c r} \approx 20 \mu \mathrm{K}$. In general, $\nu_{e f f}$ and $T_{c r}$ depend on $\theta_{0}$ and the direction of the laser field. The maximum $T_{c r}$ is reached when $\mathbf{k}$ is parallel to $\mathbf{k}_{L}$ for the most of the emitted photons. For the geometrical arrangement displayed in Fig. 2, the variation of $T_{c r}$ as a function of $\theta_{0}$ is given by Eqs. (A8) of Appendix A, and it is displayed in Fig. 3.

Let us choose parameters of Raman transitions

$$
\theta_{1}=0, \quad \theta_{2}=x, \quad \theta_{1}^{\prime}=2 x, \quad \theta_{2}^{\prime}=3 x
$$

and $T / T_{c r}=0.5$, for such case factors $S_{g e}(x)=-S_{e e}(x)$ and $S_{e g}(x)=-S_{g g}(x)$ are shown in Fig. 4(a).

One can observe the violation of BI $\left|S_{\alpha \beta}(x)\right|>2$ for $S_{g e}(x)$ and $S_{e e}(x)$, while BI are satisfied for $S_{e g}(x)$ and $S_{g g}(x)$. This situation can be inverted by choosing $\theta_{1}=0$, $\theta_{2}=-x, \theta_{1}^{\prime}=2 x$, and $\theta_{2}^{\prime}=-3 x$, so that BI will be violated for $S_{e g}(x)$ and $S_{g g}(x)$ but satisfied for $S_{g e}(x)$ and $S_{e e}(x)$. Therefore, all four states do violate BI, but the combination of angles to be used depend on the state in the pairwise fashion just described. Figure 4(b) shows the maxima of $\left|S_{\alpha \beta}\right|$ versus the normalized temperature of the atom motion found for $\theta_{1,2}, \theta_{1,2}^{\prime}$ given by Eqs. (19). The condition to vio- 


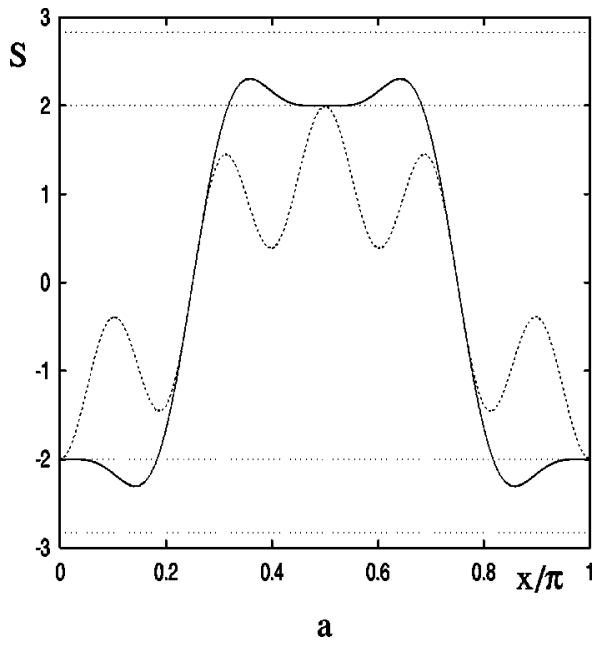

late BI for all four states (for suitable choices of Raman angles) is therefore that $T<T_{c r}$.

\section{EFFECT OF MULTIPLE SCATTERING ON BELL'S INEQUALITIES TEST}

Now we take into account the excitation of two atoms together and examine how it influences the BI violation. Let us first consider the case of only three levels in each atom shown in Fig. 5.

We examine a possibility that a photon emitted by one atom is registered, while another atom also emits a photon on $\left|e^{\prime}\right\rangle \rightarrow|e\rangle$ transition, but this photon is missed. After the excitation to $\left|e^{\prime}\right\rangle_{i}$ state the wave function of atom $i=1,2$ is given by Eqs. (2),

$$
\begin{aligned}
& \left|\Psi_{g}\right\rangle_{1}=a|g\rangle_{1}+b\left|e^{\prime}\right\rangle_{1} e^{i \mathbf{k}_{L} \delta \mathbf{r}_{1},} \\
& \left|\Psi_{g}\right\rangle_{2}=a|g\rangle_{2}-b\left|e^{\prime}\right\rangle_{2} e^{i \mathbf{k}_{L} \delta \mathbf{r}_{2}},
\end{aligned}
$$

where we suppose that conditions (11) are satisfied. If the photon $\mathbf{k}$ is emitted by one atom and registered, while another photon $\mathbf{k}^{\prime}$ is emitted by the other atom and missed, then the atoms go from $\left|e^{\prime} e^{\prime}\right\rangle$ to $|e e\rangle$ state and we have

$$
\begin{aligned}
& e^{i \mathbf{k}_{L}\left(\delta \mathbf{r}_{1}+\delta \mathbf{r}_{2}\right)}\left|e^{\prime} e^{\prime}\right\rangle \rightarrow e^{i \mathbf{k}_{L}\left(\delta \mathbf{r}_{1}+\delta \mathbf{r}_{2}\right)}\left(e^{-i \mathbf{k} \delta \mathbf{r}_{1}}\left|e e^{\prime}\right\rangle\right. \\
&\left.+e^{-i \mathbf{k} \delta \mathbf{r}_{2}}\left|e^{\prime} e\right\rangle\right)|\mathbf{k}\rangle \rightarrow f|e e\rangle\left|\mathbf{k}, \mathbf{k}^{\prime}\right\rangle \\
& f=e^{i\left(\mathbf{q} \delta \mathbf{r}_{1}+\mathbf{q}^{\prime} \delta \mathbf{r}_{2}+\varphi_{2}^{\prime}\right)}+e^{i\left(\mathbf{q} \delta \mathbf{r}_{2}+\mathbf{q}^{\prime} \delta \mathbf{r}_{1}+\varphi_{1}^{\prime}\right)}
\end{aligned}
$$

where $\mathbf{q}^{\prime}=\mathbf{k}_{L}-\mathbf{k}^{\prime}, \varphi_{i}^{\prime}$ is the phase of a missed photon emitted by atom $i=1,2$ and we suppose, as usual, $k l_{1}=k l_{2}$
FIG. 4. (a) $S_{g e}(x)=-S_{e e}(x)$ (solid line) and $S_{e g}(x)=-S_{g g}(x)$ (dashed line) at $T / T_{c r}=0.5$ and angles $\theta_{1}, \theta_{2}, \theta_{1}^{\prime}, \theta_{2}^{\prime}$ given by Eqs. (19). Bell's inequalities are violated for $S_{g e}(x)=-S_{e e}(x)$. (b) The maxima of $\left|S_{\alpha \beta}\right|$ for different $T / T_{c r}$. Dotted lines in (a) mark $|S|=2$ and $|S|=2 \sqrt{2}$.

$$
\begin{aligned}
& \frac{1}{\sqrt{N}}\left[\left(e^{i \mathbf{q} \delta \mathbf{r}_{1}}|e g\rangle-e^{i \mathbf{q} \delta \mathbf{r}_{2}}|g e\rangle\right)|\mathbf{k}\rangle+(\xi)^{1 / 2} f|e e\rangle\right. \\
& \left.\quad \times\left(\left|\mathbf{k}^{\prime}, \mathbf{k}\right\rangle+\left|\mathbf{k}, \mathbf{k}^{\prime}\right\rangle\right)\right],
\end{aligned}
$$

where $\xi=(b / a)^{2}$. Taking into account that the field state $\left|\mathbf{k}^{\prime}, \mathbf{k}\right\rangle$ is orthogonal to $\left|\mathbf{k}, \mathbf{k}^{\prime}\right\rangle$, and calculating $\left\langle|f|^{2}\right\rangle_{T}=2$, one obtains the normalizing factor $N=2[1+2 \xi]$.

After carrying out the Raman transitions, the atom states in the right part of Eq. (21) are changed in accordance with the transformation (12). Following the procedure of Sec. III we find

$$
E_{g g}\left(\theta_{i}\right)=-[1-D(T)] \sin 2 \theta_{1} \sin 2 \theta_{2}-\frac{\cos 2 \theta_{1} \cos 2 \theta_{2}}{1+2 \xi} .
$$

Figure 6 shows $S_{g g}(x)$ calculated with the help of Eqs. (16) and (22) for $T / T_{c r}=0.5, \theta_{1}, \theta_{2}, \theta_{1}^{\prime}$, and $\theta_{2}^{\prime}$ given by Eqs. (19) and various $\xi$. If the state $\left|e^{\prime}\right\rangle_{i}$ is excited by a weak "square" pulse, so that $\Omega_{g i}$ is constant during the excitation time and zero otherwise, then $\xi=\left|\Omega_{g i}\right|^{2} / \delta^{2} \ll 1$, where $\delta$ is

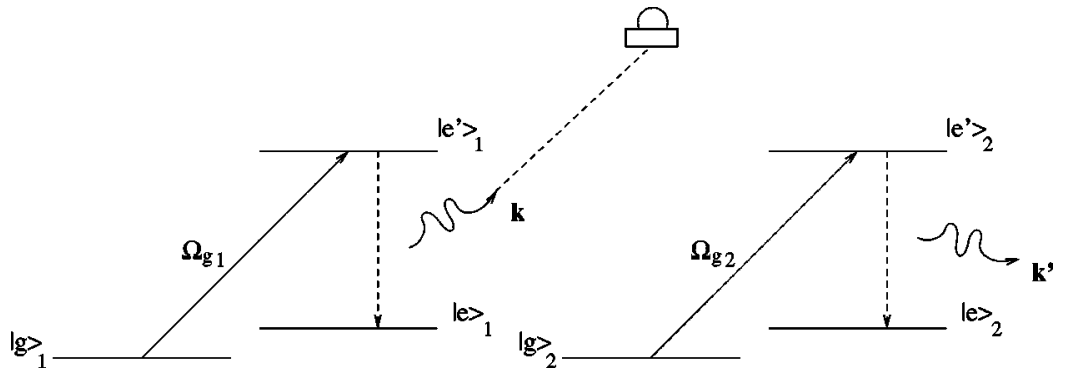

FIG. 5. This scheme represents the event where both atoms are transferred together to the $|e\rangle$ state, but one emitted photon is missed. 


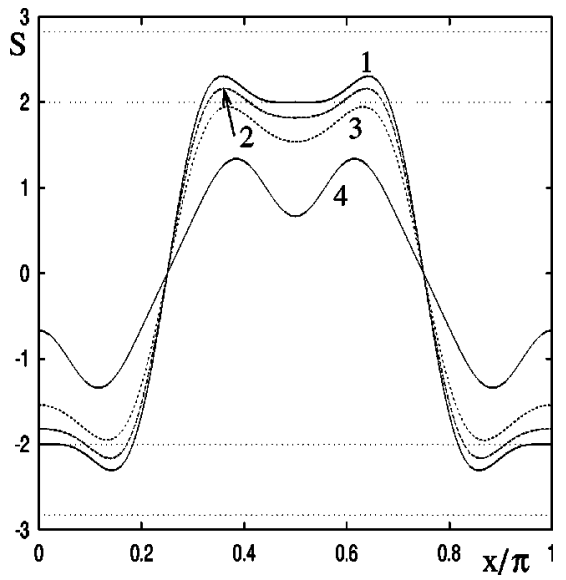

FIG. 6. Factor $S_{g g}(x)$ for Raman transition parameters given by Eqs. (19), $T / T_{c r}=0.5$ and $\xi=0$ (curve 1), $\xi=0.05$ (2), $\xi=0.15$ (3) and $\xi=1$ (4). BI violation is observed for $\xi$ up to 0.13 .

the detuning from the resonance on $|g\rangle_{i} \rightarrow\left|e^{\prime}\right\rangle_{i}$ transition. BI are violated for $\left|\Omega_{g i}\right|^{2} / \delta^{2} \leqslant 0.13$ if $T=0.5 T_{c r}$, as it can be seen on Fig. 6.

It is convenient to write the final two-atom state, taking into account simultaneous excitation of two atoms for each initial state $|\alpha \beta\rangle$, under the following matrix form:

$$
\sqrt{2 / N}\left(|\mathbf{k}\rangle \hat{B}+\left|\mathbf{k}, \mathbf{k}^{\prime}\right\rangle \hat{B}^{(2)}\right)|\alpha \beta\rangle,
$$

where the state $\left|\mathbf{k}, \mathbf{k}^{\prime}\right\rangle$ is orthogonal to $|\mathbf{k}\rangle$ and normalized to 1 , the matrix $[B]$ of the operator $\hat{B}$ is given by Eq. (10), and the matrix of the operator $\hat{B}^{(2)}$ is

$$
\left[B^{(2)}\right]=\sqrt{2 \xi}\left[\begin{array}{cccc}
0 & 0 & 0 & 1 \\
0 & 0 & 1 & 0 \\
0 & 1 & 0 & 0 \\
1 & 0 & 0 & 0
\end{array}\right]
$$

\section{BELL'S STATE MEASUREMENT}

In the preceding section we have shown that four orthogonal Bell's states can be prepared from four initial factorized states, under the condition of detecting a single photon. The reverse process, usually known as a Bell's measurement, is actually also realized using the same scheme. Here we will evaluate the efficiency of a whole sequence, including the preparation followed by the measurement-two successive clicks will be therefore required.

By carrying out the steps (1)-(3) of the procedure described in Sec. III we prepare a Bell's state $\left|B_{\alpha \beta}\right\rangle$ of two atoms. Then $\left|B_{\alpha \beta}\right\rangle$ can be projected to the pure state $|\alpha \beta\rangle$ of two atoms, or "measured," by proceeding the steps (1)-(3) with the phases of the laser fields,

$$
\varphi_{e 2}^{0}=\pi-\mathbf{k}_{L} \Delta \mathbf{r}, \quad \varphi_{g 1}^{0}=\varphi_{e 1}^{0}=0, \quad \varphi_{g 2}^{0}=-\mathbf{k}_{L} \Delta \mathbf{r} .
$$

Taking into account the multiple scattering, one arrives to the final state of two atoms and spontaneously emitted photons after the Bell's-state preparation from $|\alpha \beta\rangle$ state followed by the Bell's-state measurement,

$$
\sqrt{1 / \widetilde{N}}\left(|\widetilde{\mathbf{k}}\rangle \hat{B}+\left|\widetilde{\mathbf{k}}, \widetilde{\mathbf{k}}^{\prime}\right\rangle \hat{B}^{(2)}\right)\left(|\mathbf{k}\rangle \hat{B}+\left|\mathbf{k}, \mathbf{k}^{\prime}\right\rangle \hat{B}^{(2)}\right)|\alpha \beta\rangle,
$$

where $\widetilde{N}$ is the normalizing factor. A matrix $[\widetilde{B}]$ of an operator $\hat{\widetilde{B}}$ is $[B]^{-1}$ with $\delta \mathbf{r}_{i}$ replaced by $\delta \tilde{\mathbf{r}}_{i}$, operator $\hat{B}^{(2)}$ is the same for the Bell's-state preparation and the measurement. Since the preparation and the measurement of a Bell's state are separated in time, all field states in Eq. (25) are orthogonal to each other and the average over the atom motion yields $\left\langle\delta \mathbf{r}_{i} \cdot \delta \tilde{\mathbf{r}}_{j}\right\rangle=0, i, j=1,2$, because the atom motions on different time intervals are not correlated.

The Bell's-state measurement is not perfect due to the atom motion and the multiple scattering, so that the state (25) is, in general, a linear combination of four states (1). If a fidelity of the Bell's-state measurement is high, the probability to find atoms in $|\alpha \beta\rangle$ initial state after the measurement approaches 1 , while the probabilities to find any other atom states go to 0 . A matrix for the transformation of the vector column of states (1) after the Bell's state preparation and the measurement is

$$
\sqrt{1 / \widetilde{N}}\left([B][\widetilde{B}]+\left[B^{(2)}\right][\widetilde{B}]+[B]\left[B^{(2)}\right]+\left[B^{(2)}\right]^{2}\right) .
$$

Taking the square modulus of each element in the matrix (26) and calculating $\widetilde{N}=[1+2 \xi]^{2}$ one converts matrix (26) to a matrix of probabilities to find atoms in $|\gamma \delta\rangle$ final state starting with $|\alpha \beta\rangle$ initial state,

$$
\frac{1}{(1+2 \xi)^{2}}\left[\begin{array}{cccc}
1-f_{1}+4 \xi^{2} & 2 \xi & 2 \xi & f_{1} \\
2 \xi & 1-f_{1}+4 \xi^{2} & f_{1} & 2 \xi \\
2 \xi & f_{1} & 1-f_{1}+4 \xi^{2} & 2 \xi \\
f_{1} & 2 \xi & 2 \xi & 1-f_{1}+4 \xi^{2}
\end{array}\right]
$$

$$
f_{1}(T)=D(T)-D^{2}(T) / 2 .
$$



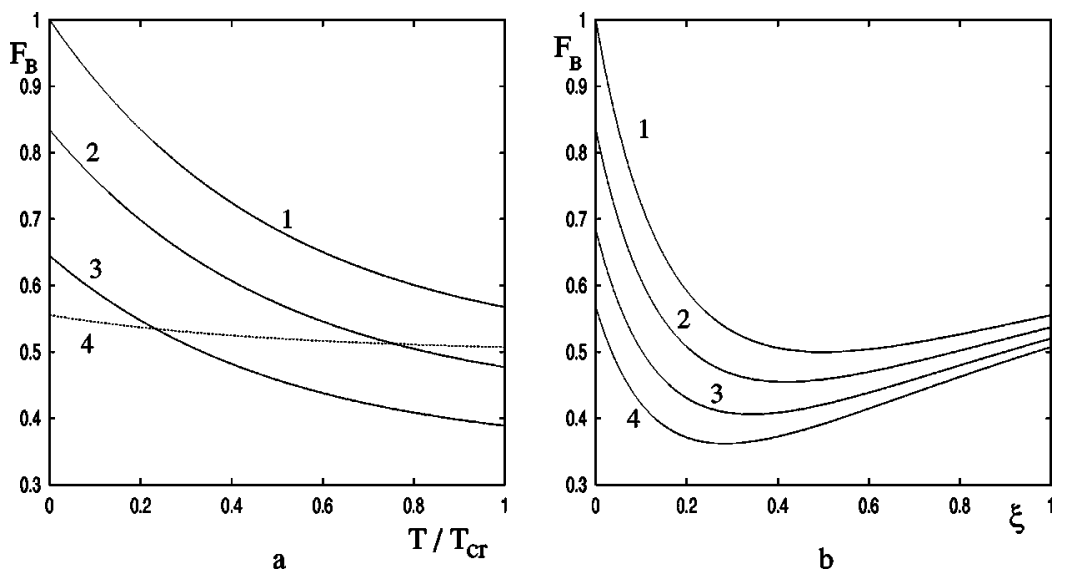

FIG. 7. (a) Fidelity $F_{B}$ for Bell's-state measurement as a function of $T / T_{c r}$ for $\xi=0$ (curve 1), $\xi=0.05$ (2), $\xi=0.15$ (3), and $\xi=1$ (4); (b) $F$ as a function of $\xi$ at $T / T_{c r}=0$ (curve 1), $T / T_{c r}$ $=0.2$ (2), $T / T_{c r}=0.5$ (3), $T / T_{c r}=1$ (4).
In the case of perfect Bell's-state preparation and the measurement, the matrix (27) has diagonal elements equal to 1 and other elements equal to 0 . Thus, we can take the diagonal element of the matrix (27) as the fidelity $F_{B}(\xi, T)$ of Bell's-state measurement,

$$
F_{B}(\xi, T)=1-\frac{4 \xi+D(T)-D^{2}(T) / 2}{(1+2 \xi)^{2}}
$$

Fidelity $F_{B}$ is shown in Fig. 7(a) as a function of $T / T_{c r}$ for various $\xi$, it is shown in Fig. 7(b) as a function of $\xi$ for various $T / T_{c r}$.

Small increase in $F_{B}$ for large $\xi \approx 1$ is because of the contribution of processes $|\alpha \alpha\rangle \rightarrow|\beta \beta\rangle \rightarrow|\alpha \alpha\rangle, \alpha \neq \beta$ grows up with $\xi$ due to the multiple photon scattering. However, this is not so important for practical cases, where $\xi \ll 1$.

\section{QUANTUM CNOT GATE}

We have shown so far that conditional Bell's-states preparation and measurement can be successfully achieved. Our result is actually more general than that, and shows that arbitrary conditional unitary transformations on two qubits can be achieved by using Raman rotations (applied locally to each atom) and the detection of a single click. In order to demonstrate this we will now show that the four Bell's-states preparation $\left|B_{\alpha \beta}\right\rangle=\hat{B}|\alpha \beta\rangle$ can be turned into a CNOT operation $\hat{C}$, described by the matrix

$$
[C]=\left[\begin{array}{llll}
1 & 0 & 0 & 0 \\
0 & 1 & 0 & 0 \\
0 & 0 & 0 & 1 \\
0 & 0 & 1 & 0
\end{array}\right] .
$$

We prove that in our case $\hat{C}=\hat{H}_{2} \hat{B} \hat{H}_{1}$, where $\hat{H}_{1,2}$ are some local (single-atom) operations and the matrix of Bell's operation $\hat{B}$ is given by Eq. (10). The CNOT operation can thus be realized by the following procedure.

(1) One of the initial states (1) of atoms is prepared.

(2) The local operation $\hat{H}_{1}$ is carried out.
(3) Atoms are excited and a spontaneously emitted photon is registered. If there are no photons registered for a time $t_{0} \gg \Gamma^{-1}$ operations (1)-(3) have to be repeated.

(4) The local operation $\hat{H}_{2}$ is carried out. Let us suppose, for a while, that atoms do not move, so that the Bell's operator is $\hat{B}_{0}$, where matrix $\left[B_{0}\right]$ is given by Eq. (10) with $\delta \mathbf{r}_{i}=\mathbf{0}$. By definition $[C]=\left[H_{1}\right]\left[B_{0}\right]\left[H_{2}\right]$, where $\left[H_{1,2}\right]$ are the matrices of local operations $\hat{H}_{1,2}$, and therefore

$$
\left[H_{1}\right]=[C]\left[H_{2}\right]^{-1}\left[B_{0}\right]^{-1} .
$$

Taking the matrix $\left[\mathrm{H}_{2}\right]^{-1}$ as a general local transformation for two-level atom, inserting it in Eq. (30) with the requirement that the matrix product on the right of Eq. (30) should be a local transformation, we obtain

$$
\begin{gathered}
{\left[H_{1}\right]=\frac{1}{2}\left[\begin{array}{cccc}
i & i & -i & -i \\
-1 & 1 & 1 & -1 \\
i & i & i & i \\
-1 & 1 & -1 & 1
\end{array}\right],} \\
{\left[H_{2}\right]=\frac{1}{\sqrt{2}}\left[\begin{array}{cccc}
0 & -1 & 0 & -i \\
i & 0 & 1 & 0 \\
0 & -1 & 0 & -i \\
-i & 0 & 1 & 0
\end{array}\right] .}
\end{gathered}
$$

Details of the procedure of determining of $\left[H_{1,2}\right]$ are given in Appendix B. As it can be seen from Eqs. (B9) and (B10) of Appendix $\mathrm{B}$, operation $\hat{H}_{1}$ is the phase transformation $|g\rangle_{2} \rightarrow i|g\rangle_{2}$, after which the Raman transition (12) with $\theta_{1}$ $=\pi / 4, \theta_{2}=-\pi / 4$ is carried out. Operation $\hat{H}_{2}$ starts with the Raman transition (12) with $\theta_{1}=-\pi / 4, \theta_{2}=-\pi / 2$ after which one makes the phase transformations $|e\rangle_{1} \rightarrow-i|e\rangle_{1}$, $|g\rangle_{2} \rightarrow-i|g\rangle_{2}$.

Now we take into account the atom motion, the simultaneous excitation of two atoms and find a fidelity of the CNOT operation. We suppose that $\hat{H}_{1,2}$ transformations are much faster than a period of the atom motion in the trap, in such case $\hat{H}_{1,2}$ does not depend at all on the atom motion. Indeed, 

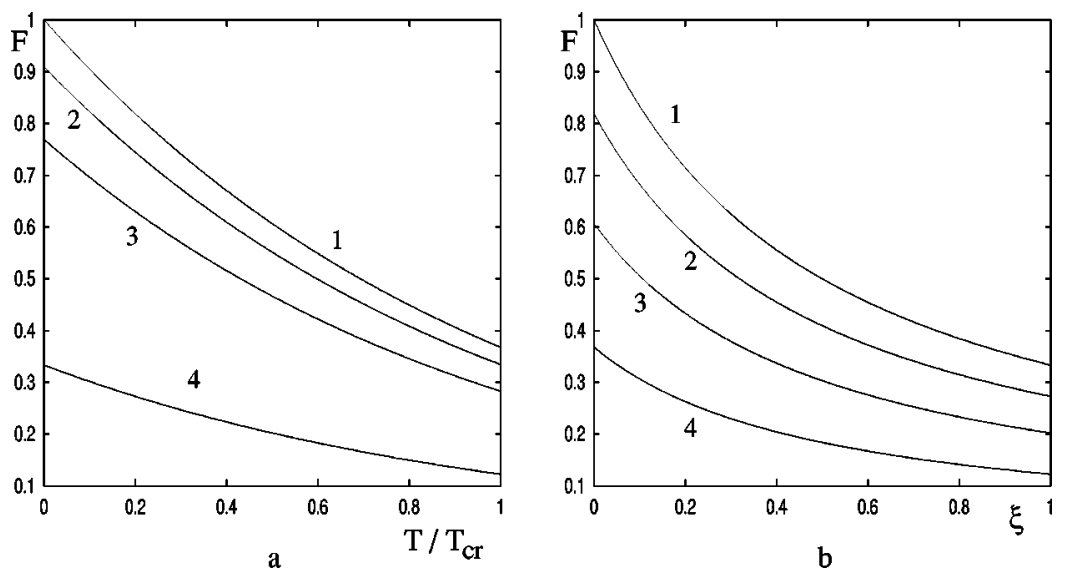

FIG. 8. (a) Fidelity $F$ for quantum CNOT gate as a function of $T / T_{c r}$ for $\xi=0$ (curve 1 ), $\xi$ $=0.05$ (2), $\xi=0.1$ (3) and $\xi=1$ (4); (b) $F$ as a function of $\xi$ at $T / T_{c r}=0$ (curve 1), $T / T_{c r}=0.2$ (2), $T / T_{c r}=0.5$ (3), $T / T_{c r}=1$ (4). by carrying out a fast local operation with atom $i$, one can choose the origin of the coordinate system in that atom, which means $\mathbf{r}_{i 0}+\delta \mathbf{r}_{0} \equiv \mathbf{0}$. Thus, using formula (10) with $\delta \mathbf{r}_{i} \neq \mathbf{0}$ and Eq. (23) we obtain an operator $\hat{C}$ of a nonperfect CNOT transformation,

$$
\hat{C}=\sqrt{2 / N}\left(|\mathbf{k}\rangle \hat{C}^{(1)}+\left|\mathbf{k}, \mathbf{k}^{\prime}\right\rangle \hat{C}^{(2)}\right)
$$

where matrices of operators $\hat{C}^{(1,2)}$ are

$$
\left[C^{(1)}\right]=\left[H_{1}\right][B]\left[H_{2}\right], \quad\left[C^{(2)}\right]=\left[H_{1}\right]\left[B^{(2)}\right]\left[H_{2}\right] .
$$

The matrices $\left[H_{1,2}\right]$ are given by Eqs. (31), and matrices $[B],\left[B_{2}\right]$ are given by Eqs. (10),(24), respectively.

We can now build matrices $\left[\left|C^{(1,2)}\right|^{2}\right]$, whose elements are the square modulus of respective elements of $\left[C^{(1,2)}\right]$, and calculate the matrix $\left[C_{p}\right]$ of probabilities to find atoms in $|\gamma \delta\rangle$ state after the CNOT operation, while $|\alpha \beta\rangle$ was the initial atom state,

$$
\begin{aligned}
{\left[C_{p}\right] } & =\frac{2}{N}\left\{\left[\left|C^{(1) \mid}\right|^{2}\right]+\left[\left|C^{(1)}\right|^{2}\right]\right\} \\
& =\frac{1}{2}\left[\begin{array}{cccc}
1+F & 1-F & 0 & 0 \\
1-F & 1+F & 0 & 0 \\
0 & 0 & 1-F & 1+F \\
0 & 0 & 1+F & 1-F
\end{array}\right],
\end{aligned}
$$

where

$$
F=\frac{1-D(T)}{1+2 \xi},
$$

$0<F<1$ is the fidelity of the CNOT operation (32). The value of $F$ is shown in Fig. 8(a) as a function of $T / T_{c r}$ for various $\xi$, and it is shown in Fig. 8(b) as a function of $\xi$ for various $T / T_{c r}$.

\section{DISCUSSION}

An important problem in the experimental demonstration of the conditional quantum CNOT gate operation is the suppression of the atom motion, which can be done by cooling atoms in the traps down to the temperatures of few $\mu \mathrm{K}$, or by increasing the atom oscillation frequencies. This can be done, for example, by using standing-wave trapping fields, that separate a trapped potential into several narrow wells with oscillation frequencies much higher than the present 50 $\mathrm{kHz}$ obtained for the longitudinal motion in a tightly focused beam. Multiple scattering gives a rather small contribution, as long as the saturation parameter remains below a few percents. An important characteristics of the fidelity of the CNOT gate operation is the decoherence parameter $D(T)$. For a reliable theoretical determination of $D(T)$, one needs to know accurately the trapping potential. However, before doing experiments on BI violation, $D(T)$ can also be determined experimentally by looking at the interference fringes on the light emitted by the two atoms, irradiated on a closed transition [20].

Neutral atoms in a dipole trap are not the only candidates for implementing our conditional CNOT gate. In principle, the gate can be realized with other resonant objects such as, for example, trapped ions, or quantum dot molecules (QDMs) incorporated in a solid matrix [21,22]. Each QDM consists in two closely positioned quantum dots with the ground state of each dot split into two or more close states. The advantages of QDMs are their fixed positions in the matrix and the possiblity to prepare the initial states electronically. The difficulty, however, is in providing the coherence during the gate operation, which is quickly destroyed by the electron-phonon interaction.

The proposed simple scheme can be generalized straightforwardly to more complicated schemes with many elementary gates, which may be called "integrated conditional quantum logic blocks," or ICQLB. They can be constructed by the increase of the number of atoms and (or) the number of ground states available in a single atom. There are $p^{n}$ initial states of $n$ atoms, if an identical photon can be emitted on transitions to $p$ different ground states. However, because the photon observation process is not Hermitian, the maximum number $N(n, p)$ of obtained orthogonal Bell's states is, in general, less than $p^{n}$, though $N(n, p)$ increases with $n$ and $p$. For example, only seven orthogonal Bell's states are possible for three atoms with the level scheme of Fig. 1, for any choice of phases of laser fields. Determination of $N(n, p)$ is an important question for the theoretical modeling of ICQLB. 
Another theoretical problem is how to find local transformations, which convert $N(n, p) \times N(n, p)$ matrix of the generalized Bell's transformation, obtained by the photon observation procedure, to the matrix of desirable logic transformation. The procedure of Appendix B can be generalized, in principle, to higher-dimensional cases, however it seems too cumbersome, and the development of a simpler procedure would be quite helpful. We underline that even complicated ICQLB operate in the same five steps as the CNOT gate described above. The step (1) is the preparation of initial $N(n, p)$ states of atoms; (2) includes local transformations; (3) is the excitation of atoms by weak resonant fields, repeated maybe several times until a spontaneously emitted photon is registered; (4) is another local transformation; (5) is the determination of final populations of atomic states. Each step can be carried out simultaneously for all atoms together, so that the operation time of ICQLB is not much longer than for the elementary CNOT gate. The increase in the operation time of complicated ICQLB can happen, however, because the probability for $n$ atoms to emit more than one photon increases with $n$, so that lower intensities of the exciting fields are required in order to avoid multiple scattering.

Finally, using conditional gates in a quantum computer requires to "store them aside" once they are known to work, and to teleport them in the calculation at a later stage [12]. A similar scheme might be possible with atomic qubits, as proposed in Ref. [23]. Though we do not propose here a specific way to make the present scheme scalable, a further direction of research is clearly to study up to which point quantum computations may be realized using conditional logical elements such as the ones described above.

\section{APPENDIX A}

Here we show that $D(T)$ can be given by Eq. (18) within some approximations, and we estimate the critical temperature $T_{c r}$. We consider an atom in the trapping potential as a harmonic oscillator, with a deviation from the equilibrium position given by $\delta \mathbf{r}_{i}=\left\{\delta r_{x}, \delta r_{y}, \delta r_{z}\right\}$. Then $\left\langle e^{i \mathbf{q} \delta r_{i}}\right\rangle_{T}$ $=e^{-\left\langle\left(\mathbf{q} \delta \mathbf{r}_{i}\right)^{2}\right\rangle_{T} / 2}$, which is the consequence that the thermal fluctuations of the position of a harmonic oscillator are described by the Gaussian distribution function, and therefore

$$
1-D(T) \equiv\left\langle e^{i \mathbf{q}\left(\delta \mathbf{r}_{1}-\delta \mathbf{r}_{2}\right)}\right\rangle=\left\langle e^{-\left\langle(\mathbf{q} \delta \mathbf{r})^{2}\right\rangle_{T}}\right\rangle_{\mathbf{q}}
$$

In the coordinate system shown in Fig. 2, $k_{x}=k \sin \theta \cos \varphi$, $k_{y}=k \sin \theta \sin \varphi, k_{z}=k \cos \theta$ and $k_{L x} \approx k, k_{L y}=k_{L z}=0$, so that

$$
\begin{aligned}
\left\langle(\mathbf{q} \delta \mathbf{r})^{2}\right\rangle_{T}= & k^{2}\left[(1-\sin \theta \cos \varphi)^{2}\left\langle\delta r_{x}^{2}\right\rangle_{T}+\sin ^{2} \theta \sin ^{2} \varphi\left\langle\delta r_{y}^{2}\right\rangle_{T}\right. \\
& \left.+\cos ^{2} \theta\left\langle\delta r_{z}^{2}\right\rangle_{T}\right] .
\end{aligned}
$$

For the one-dimensional quantum harmonic oscillator with the mass $m_{a t}$, which oscillates along the axes $\xi$ with the frequency $\omega_{\xi}, \xi=x, y, z$ in the thermal equilibrium

$$
\left\langle\delta r_{\xi}^{2}\right\rangle_{T}=\frac{\hbar}{2 m_{a t} \omega_{\xi}} \operatorname{coth}\left(\frac{\hbar \omega_{\xi}}{2 k_{B} T}\right) \approx \frac{k_{B} T}{m_{a t} \omega_{\xi}^{2}},
$$

at $\hbar \omega_{\xi} /\left(k_{B} T\right) \ll 1$. Taking $\omega_{x}=\omega_{y}=2 \pi \nu_{\perp}$ and $\omega_{z}=2 \pi \nu_{\|}$ we obtain

$$
\begin{aligned}
\left\langle(\mathbf{q} \delta \mathbf{r})^{2}\right\rangle_{T}= & k^{2} \frac{k_{B} T}{2 \pi^{2} m_{a t}}\left[\left(1+\sin ^{2} \theta-2 \sin \theta \cos \varphi\right) \frac{1}{\nu_{\perp}^{2}}\right. \\
& \left.+\cos ^{2} \theta \frac{1}{\nu_{\|}^{2}}\right] .
\end{aligned}
$$

The average

$$
\langle u(\theta, \varphi)\rangle_{\mathbf{q}}=\int_{0}^{2 \pi} d \varphi \int_{0}^{\theta_{0}} \sin \theta d \theta \mathcal{P}(\theta, \varphi) u(\theta, \varphi),
$$

where $u(\theta, \varphi)$ is some function and

$$
\mathcal{P}(\theta, \varphi)=C_{0}\left(1-\sin ^{2} \theta \cos ^{2} \varphi\right)
$$

is the probability that a photon is emitted within the optical system with a direction $\mathbf{k}$. The normalizing constant $C_{0}$ is given by

$$
\begin{aligned}
\frac{1}{C_{0}} & =\int_{0}^{2 \pi} d \varphi \int_{0}^{\theta_{0}} \sin \theta d \theta\left(1-\sin ^{2} \theta \cos ^{2} \varphi\right) \\
& =\frac{4 \pi}{3}\left[1-\frac{1}{4}\left(3 \cos \theta_{0}+\cos ^{3} \theta_{0}\right)\right] .
\end{aligned}
$$

Eq. (A6) is obtained from the relation

$$
\mathcal{P}(\theta, \varphi)=C_{0} \sum_{\lambda=1,2}\left[\mathbf{d} \cdot \mathbf{e}_{\lambda}(\mathbf{k} / k)\right]^{2}=C_{0}\left[1-(\mathbf{d} \cdot \mathbf{k})^{2} / k^{2}\right],
$$

where $\mathbf{e}_{\lambda}(\mathbf{k})$ is the unit wave vector along one of the two possible polarizations $\lambda=1,2$ of a photon, and $\mathbf{d}$ is a unit wave vector along the $\pi$-polarized atomic dipole ( $x$ axis on Fig. 2).

Thus, in order to find $D(T)$ one has to insert Eq. (A4) into Eq. (A1) and calculate $\langle\cdots\rangle_{\mathbf{q}}$ from Eq. (A5). For a highinput aperture of the optical system, this procedure can hardly lead to an analytical result, and we use the approximation

$$
\left\langle e^{-\left\langle(\mathbf{q} \delta \mathbf{r})^{2}\right\rangle_{T}}\right\rangle_{\mathbf{q}} \approx e^{-\left\langle\left\langle(\mathbf{q} \delta \mathbf{r})^{2}\right\rangle_{T}\right\rangle_{\mathbf{q}}}
$$

which improves as $D(T)$ gets smaller. Thus, we obtain finally $D(T)=1-e^{-T / T_{c r}\left(\theta_{0}\right)}$, that is Eq. (18) where

$$
k_{B} T_{c r}\left(\theta_{0}\right)=\frac{h^{2} \nu_{e f f}^{2}\left(\theta_{0}\right)}{2 E_{R}}, \frac{1}{\nu_{e f f}^{2}\left(\theta_{0}\right)}=\frac{A_{\|}\left(\theta_{0}\right)}{\nu_{\|}^{2}}+\frac{A_{\perp}\left(\theta_{0}\right)}{\nu_{\perp}^{2}},
$$

$$
A_{\perp}\left(\theta_{0}\right)=1+\frac{4 \pi C_{0}\left(\theta_{0}\right)}{5}\left(1-\frac{5 \cos \theta_{0}-\cos ^{5} \theta_{0}}{4}\right),
$$




$$
A_{\|}\left(\theta_{0}\right)=\frac{8 \pi C_{0}\left(\theta_{0}\right)}{15}\left(1-\frac{5 \cos ^{3} \theta_{0}+3 \cos ^{5} \theta_{0}}{8}\right),
$$

and $C_{0}\left(\theta_{0}\right)$ is determined by Eq. (A7).

\section{APPENDIX B:}

In this paper we use the relation $[A B]=[B][A]$, where $[A B],[A]$, and $[B]$ are the matrices of the operators $\hat{A} \hat{B}, \hat{A}$, and $\hat{B}$, respectively, acting on the wave functions from the vector column (1). Let us prove this relation. We name the wave functions from the vector column (1) as $\left|\psi_{i}\right\rangle, i$ $=1, \ldots, 4$ and use the relation $\hat{L}\left|\psi_{i}\right\rangle=\sum_{k=1}^{4} L_{i k}\left|\psi_{k}\right\rangle$, where $L_{i k}$ is the matrix element of the matrix $[L]$ of the operator $\hat{L}$ in the basis $\left\{\left|\psi_{i}\right\rangle\right\}$. So that we can write

$$
\begin{aligned}
\hat{A} \hat{B}\left|\psi_{i}\right\rangle & =\sum_{k=1}^{4}[B]_{i k} \hat{A}\left|\psi_{k}\right\rangle=\sum_{l=1}^{4}\left(\sum_{k=1}^{4}[B]_{i k}[A]_{k l}\right)\left|\psi_{l}\right\rangle \\
& =\sum_{l=1}^{4}[A B]_{i l}\left|\psi_{l}\right\rangle,
\end{aligned}
$$

where $[A]_{k l},[B]_{i k}$, and $[A B]_{i l}$ are the elements of $[A]$, $[B]$, and $[A B]$, respectively, which proves $[A B]_{i l}$ $=\sum_{k=1}^{4}[B]_{i k}[A]_{k l}$ and therefore $[A B]=[B][A]$.

Now we find the matrix of the local transformations, which mixes the states $|g\rangle_{i}$ and $|e\rangle_{i}$ of the two-level atom $i=1,2$. Such transformation is necessary for the calculation of the probabilities (13) and in order to solve Eq. (30). General local transformations can be written in the form

$$
\begin{gathered}
|g\rangle_{i} \rightarrow\left(c_{i} e^{i \phi_{g i}}|g\rangle_{i}-s_{i} e^{i \phi_{e i}}|e\rangle_{i}\right) e^{i \xi_{g i}}, \\
|e\rangle_{i} \rightarrow\left(c_{i} e^{i \phi_{e i}}|e\rangle_{i}+s_{i} e^{i \phi_{g i}}|g\rangle_{i}\right) e^{i \xi_{e i}}
\end{gathered}
$$

They consist of the phase transformation which changes the phase of the state $|\alpha\rangle_{i} \rightarrow e^{i \xi_{\alpha i}}|\alpha\rangle_{i}$, the Raman transition given by Eq. (12) with $c_{i} \equiv \cos \left(\theta_{i}\right), s_{i} \equiv \sin \left(\theta_{i}\right)$, and another phase transformation $|\alpha\rangle_{i} \rightarrow e^{i \phi_{\alpha i}}|\alpha\rangle_{i}$. For our purposes, however, it is enough to consider transformations (B1) (or their reverse) with $\phi_{\alpha i}=0$. The matrix of the local transformations carried out with two atoms is, therefore,

$$
\begin{aligned}
{\left[L\left(\theta_{i}, \xi_{\alpha i}\right)\right]=[} & \left.M\left(\xi_{\alpha i}\right)\right]\left[R\left(\theta_{i}\right)\right] \equiv\left[M\left(\xi_{\alpha i}\right)\right] \\
& \times\left[\begin{array}{cccc}
c_{1} c_{2} & -c_{1} s_{2} & -s_{1} c_{2} & s_{1} s_{2} \\
c_{1} s_{2} & c_{1} c_{2} & -s_{1} s_{2} & -s_{1} c_{2} \\
s_{1} c_{2} & -s_{1} s_{2} & c_{1} c_{2} & -c_{1} s_{2} \\
s_{1} s_{2} & s_{1} c_{2} & c_{1} s_{2} & c_{1} c_{2}
\end{array}\right],
\end{aligned}
$$

where

$$
\begin{aligned}
{\left[M\left(\xi_{\alpha i}\right)\right] } & \equiv\left[M\left(\xi_{g 1}, \xi_{e 1}, \xi_{g 2}, \xi_{e 2}\right)\right] \\
& =\operatorname{diag}\left\{e^{i \xi_{g g}}, e^{i \xi_{g e}}, e^{i \xi_{e g}}, e^{i \xi_{e e}}\right\}, \quad \xi_{\alpha \beta} \equiv \xi_{\alpha 1}+\xi_{\beta 2},
\end{aligned}
$$

is the diagonal matrix of the phase transformation and $\left[R\left(\theta_{i}\right)\right] \equiv\left[R\left(\theta_{1}, \theta_{2}\right)\right]$ is the matrix of the Raman transformation (12) used in the calculations of the probabilities (13).

In order to solve Eq. (30), we take $\left[\mathrm{H}_{2}\right]^{-1}$ $=\left[L\left(\theta_{i}, \xi_{\alpha i}\right)\right]$, insert it into Eq. (30) and find where $\left[M_{1}\left(\xi_{\alpha i}\right)\right]$ is a diagonal matrix obtained by interchanging the two last elements of $\left[M\left(\xi_{\alpha i}\right)\right]$.

Our goal is to determine $\theta_{i}$ and $\xi_{\alpha i}$, such that the matrix given by Eq. (B4) can be represented as

$$
\left[H_{1}\right]=\left[L\left(\widetilde{\theta}_{i}, \widetilde{\xi}_{\alpha i}\right)\right]
$$

with some $\widetilde{\theta}_{1,2}, \widetilde{\xi}_{\alpha i}$. By comparing the matrices given by Eqs. (B2) and (B4) we see that Eq. (B5) can be true only if $\left|c_{i}\right|=\left|s_{i}\right|$, that is when $\theta_{i}= \pm \pi / 4, \theta_{i}= \pm 3 \pi / 4$; or when $c_{i}$ $=0$ or $s_{i}=0$, while $\left|c_{j}\right|=\left|s_{j}\right|, j \neq i$. We choose $\theta_{1}=\pi / 4$, $\theta_{2}=\pi / 2$, from which we get $c_{1}=s_{1}=1 \sqrt{2}, c_{2}=0, s_{2}-1$, and therefore

$$
\left[H_{1}\right]=\left[\begin{array}{cccc}
e^{i \xi_{g g}} & e^{i \xi_{g g}} & -e^{i \xi_{g g}} & -e^{i \xi_{g g}} \\
-e^{i \xi_{g e}} & e^{i \xi_{g e}} & e^{i \xi_{g e}} & -e^{i \xi_{g e}} \\
e^{i \xi_{e e}} & e^{i \xi_{e e}} & e^{i \xi_{e e}} & e^{i \xi_{e e}} \\
e^{i \xi_{e g}} & -e^{i \xi_{e g}} & e^{i \xi_{e g}} & -e^{i \xi_{e g}}
\end{array}\right]
$$

We can see now that the matrix $\left[L\left(\widetilde{\theta}_{i}, \widetilde{\xi}_{\alpha i}\right)\right]$ is very similar to the matrix (B6) if we take $\widetilde{\theta}_{1}=\pi / 4$ and $\widetilde{\theta}_{2}=-\pi / 4$, so that

$$
\left[L\left(\pi / 4,-\pi / 4, \widetilde{\xi}_{\alpha i}\right)\right]=\left[\begin{array}{cccc}
e^{i \tilde{\xi}_{g g}} & e^{i \tilde{\xi}_{g g}} & -e^{i \tilde{\xi}_{g g}} & -e^{i \tilde{\xi}_{g g}} \\
-e^{i \tilde{\xi}_{g e}} & e^{i \tilde{\xi}_{g e}} & e^{i \tilde{\xi}_{g e}} & -e^{i \tilde{\xi}_{g e}} \\
e^{i \tilde{\xi}_{e g}} & e^{i \tilde{\xi}_{e g}} & e^{i \tilde{\xi}_{e g}} & e^{i \tilde{\xi}_{e g}} \\
-e^{i \tilde{\xi}_{e e}} & e^{i \tilde{\xi}_{e e}} & -e^{i \tilde{\xi}_{e e}} & e^{i \tilde{\xi}_{e e}}
\end{array}\right]
$$


with

$$
\widetilde{\xi}_{\alpha \beta} \equiv \widetilde{\xi}_{\alpha 1}+\widetilde{\xi}_{\beta 2} .
$$

Apart from the notations for phases, the only difference between the matrices in Eqs. (B7) and (B6) is the opposite signs of the elements in the last rows. The simplest way to eliminate this difference by choosing $\widetilde{\xi}_{e e}=\pi$ is not permitted by relations (B8). However, by the examination of Eqs. (B6) and (B7) one can see that they are equivalent for $\xi_{e g}=\pi$, $\xi_{e e}=\xi_{g g}=\pi / 2, \quad \xi_{g e}=0$, and $\widetilde{\xi}_{e g}=\widetilde{\xi}_{g g}=\pi / 2, \quad \widetilde{\xi}_{g e}=\widetilde{\xi}_{e e}=0$. Such a choice does not contradict with Eqs. (B3) and (B8), it corresponds to $\xi_{g 1}=0, \quad \xi_{g 2}=\pi / 2, \quad \xi_{e 1}=\pi / 2, \quad \xi_{e 2}=0$ and $\widetilde{\xi}_{g 1}=0 \widetilde{\xi}_{g 2}=\pi / 2, \widetilde{\xi}_{e 1}=\widetilde{\xi}_{e 2}=0$. Inserting such values of $\widetilde{\xi}_{\alpha \beta}$ and $\widetilde{\theta}_{1}=\pi / 4, \widetilde{\theta}_{2}=-\pi / 4$ into Eqs. (B7) and (B5) we find

$$
\left[H_{1}\right]=[M(0,0, \pi / 2,0)][R(\pi / 4,-\pi / 4)] .
$$

Otherwise, the matrix $\left[\mathrm{H}_{2}\right]^{-1}$ is, by definition, given by Eqs.
(B2). Inserting there $\theta_{1}=\pi / 4, \theta_{2}=\pi / 2$, and the values of $\xi_{\alpha i}$ given above we obtain

$$
\left[H_{2}\right]=[R(-\pi / 4,-\pi / 2)][M(0,-\pi / 2,-\pi / 2,0)],
$$

where we take into account that $[R(\pi / 4, \pi / 2)]^{-1}$ $=[R(-\pi / 4,-\pi / 2)] \quad$ and $\quad[M(0, \pi / 2, \pi / 2,0)]^{-1}=[M(0$, $-\pi / 2,-\pi / 2,0)]$. The matrices $\left[H_{1,2}\right]$ satisfying Eq. (30) are given explicitly by Eqs. (31). We note that the matrices given by Eqs. (B9) and (B10) are not the only ones which satisfy Eq. (30), but other possible ones will have a similar form.

\section{ACKNOWLEDGMENTS}

This work was supported by the European IST/FET project QUBITS and by the European IHP network QUEST. I.P. is also grateful to the Russian Foundation for Basic Research, Grant No. 01-02-17330, for support.
[1] A. Steane et al., Phys. Rev. A 62, 042305 (2000).

[2] T. Calarco et al., J. Mod. Opt. 47, 2137 (2000).

[3] G.K. Brennen, I.H. Deutsch, and P.S. Jessen, Phys. Rev. A 61, 062309 (2000).

[4] D. Jaksch et al., Phys. Rev. Lett. 85, 2208 (2000).

[5] I.E. Protsenko, G. Reymond, N. Schlosser, and P. Grangier, Phys. Rev. A 65, 052301 (2002).

[6] S.B. Zheng and G.C. Guo, Phys. Rev. Lett. 85, 2392 (2000).

[7] C.A. Sackett et al., Nature (London) 404, 256 (2000).

[8] A. Rauschenbeutel et al., Phys. Rev. Lett. 83, 5166 (1999).

[9] S. Osnaghi et al., Phys. Rev. Lett. 87, 037902 (2001).

[10] D. Kielpinski, C. Monroe, and D.J. Wineland, Nature (London) 417, 709 (2002).

[11] M. Greiner, O. Mandel, T. Esslinger, T.W. Haensch, and I. Bloch, Nature (London) 415, 39 (2002).

[12] E. Knill, R. Laflamme, and G.J. Milburn, Nature (London) 409, 46 (2001).

[13] R.H. Dicke (unpublished); see also W.M. Itano et al., in Pro- ceedings of the International Conference on Lasers '93, Lake Tahoe, 1993, edited by V.J. Corcoran and T.A. Goldman (STS Press, McLean, VA, 1994), pp. 412-419.

[14] C. Cabrillo, J.I. Cirac, P. Garcia-Fernandez, and P. Zoller, Phys. Rev. A 59, 1025 (1999).

[15] N. Schlosser, G. Reymond, I. Protsenko, and P. Grangier, Nature (London) 411, 1024 (2001).

[16] N. Schlosser, G. Reymond, and P. Grangier, Phys. Rev. Lett. 89, 023005 (2002).

[17] J.H. Eberly, Am. J. Phys. 70, 276 (2002).

[18] J.S. Bell, in Foundations of Quantum Mechanics, edited by B. d'Espagnat (Academic, New York, 1972).

[19] A. Aspect, P. Grangier, and G. Roger, Phys. Rev. Lett. 49, 91 (1982).

[20] W.M. Itano et al., Phys. Rev. A 57, 4176 (1998).

[21] G. Wilfred et al., Jpn. J. Appl. Phys., Part 1 40, 2100 (2001).

[22] T.H. Oosterkamp et al., Nature (London) 395, 873 (1998).

[23] A.S. Sorensen and K. Moelmer, e-print quant-ph/0206142. 